\title{
Flecainide-Induced Torsade de Pointes Successfully Treated with Intensive Pharmacological Therapy
}
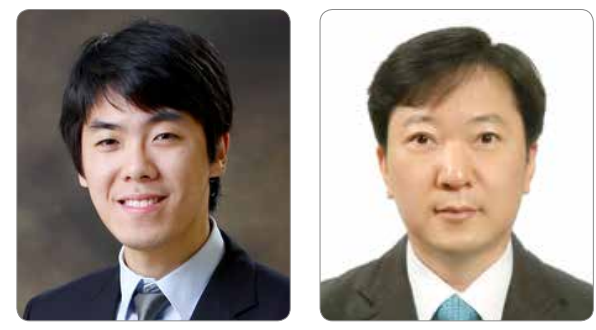

Hae Won Jung, MD, Jae-Jin Kwak, $M D$, June Namgung MD, PhD

Division of Cardiology, Department of Internal Medicine, Vision 21 Cardiac \& Vascular Center, Inje University Ilsan Paik Hospital, Goyang, Republic of Korea

Received: February 25, 2016

Revision Received: May 2, 2016

Accepted: June 29, 2016

Correspondence: June Namgung, MD, PhD

Division of Cardiology, Department of Internal Medicine, Vision 21 Cardiac \& Vascular Center, Inje University Ilsan Paik Hospital, 170 Juhwa-ro,

Ilsanseo-gu, Goyang-si, Gyeonggi-do 10380, Republic of Korea

Tel: +82-10-2974-7540

Fax: +82-31-914-7644

E-mail: jnamgung@paik.ac.kr

Copyright () 2016 The Official Journal of Korean Heart Rhythm Society Editorial Board and MMK

Communications Limited

\begin{abstract}
Flecainide acetate is a potent class IC anti-arrhythmic drug with a major sodium channel blocking effect. Flecainide toxicity can cause myocardial impairment and precipitate circulatory collapse. It may also result in life-threatening arrhythmia, although cases of flecainide-induced torsades de pointes are rare. Furthermore, the electrical and hemodynamic deteriorations observed during flecainide toxicity may not respond to conventional treatments. In the present study, we report the case of a 20-year-old Korean man with flecainide poisoning, who presented with hypotension. The patient was successfully treated with sodium bicarbonate, amiodarone, $\mathrm{MgSO}_{4}$, and lidocaine, with no recourse to extracorporeal therapy. Although there is no standard therapy for flecainide toxicity, this report demonstrates that intensive pharmacological treatment is beneficial in cases of flecainide overdose.
\end{abstract}

Key Words: - Flecainide - Arrhythmia - Drug Toxicity

\section{Introduction}

Flecainide is a class IC anti-arrhythmic drug that acts by blocking the fast inward sodium channels during phase 0 of action potential. It is used to treat both supraventricular and ventricular arrhythmias. However, flecainide is also a pro-arrhythmic agent, and overdose may cause nausea, vomiting, hypotension, bradycardia, varying degrees of atrioventricular block, tachyarrhythmias (such as torsades de pointes $[\mathrm{TdP}]$ ), and sustained ventricular tachycardia. These symptoms may result in rapid-onset hypotension, with an associated mortality rate as high as $10 \%$. The management of flecainide overdose includes supportive and pharmacological measures; however, difficult cases have been successfully treated with extracorporeal membrane oxygenation (ECMO) and intra-aortic balloon pumps. The authors present a case of a patient who visited their hospital with flecainide intoxication and life-threatening arrhythmias, including TdP, ventricular tachycardia, and ventricular fibrillation. The patient was successfully treated with intensive pharmacological therapy.

\section{Case}

A 20-year-old man who attempted to commit suicide by ingesting approximately $5,000 \mathrm{mg}$ of flecainide presented to the emergency department one hour after the ingestion. The patient 


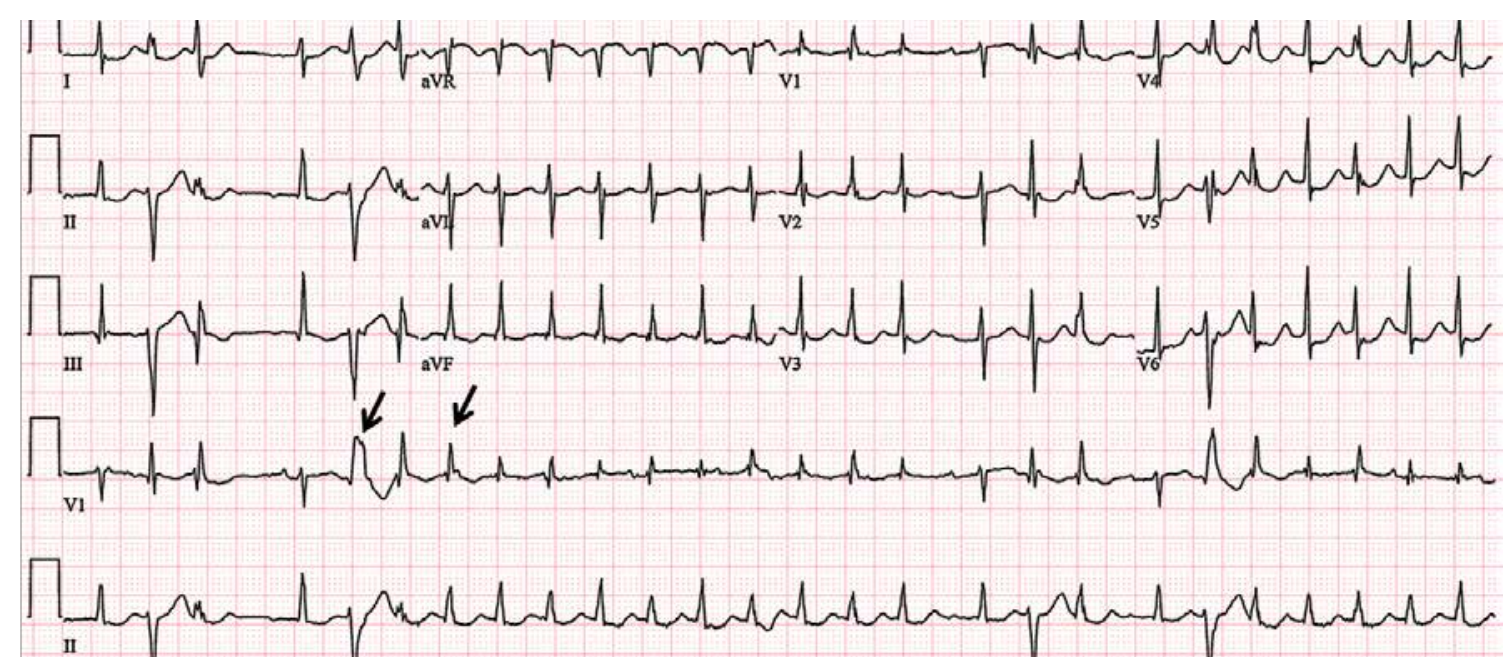

Figure 1. Initial twelve-lead electrocardiogram (ECG) upon arrival in the emergency department. The ECG showed marked QRS widening and an irregular rhythm, with a rate of 40 to 55 beats/min. The QRS duration is $168 \mathrm{msec}$ and corrected QT interval is $602 \mathrm{msec}$.

had been taking flecainide as a "pill in the pocket" for the treatment of supraventricular tachycardia over the previous month. Before arrival at Ilsan Paik hospital, the patient had a bout of vomiting and grand mal seizure in the national 119-rescue services ambulance. Two generalized seizures were also noted during his transport to the hospital. Upon arrival at the emergency department, the patient was semi-comatose, with a Glasgow Coma Scale score of 4 .

His blood pressure was $44 / 23 \mathrm{mmHg}$, and the electrocardiogram (ECG) showed an irregular, wide complex bradycardia with a heart rate of 40 to 55 BPM (Figure 1). The initial treatment included intravenous (IV) administration of normal saline and dopamine. Despite initial management, the patient had an episode of irregular, wide QRS tachycardia as well as consecutive pulseless ventricular fibrillations (Figure 2); hence, cardiopulmonary resuscitation (CPR) was started. The initial arterial blood gas showed a $\mathrm{pH}$ of 7.241; $\mathrm{pCO}_{2}$ was $32.2 \mathrm{mmHg}$, $\mathrm{pO}_{2} 117.9 \mathrm{mmHg}$, and $\mathrm{HCO}_{3}-13.5 \mathrm{mmol} / \mathrm{L}$. Chemical screens revealed blood urine nitrogen (BUN) level of $12 \mathrm{mg} / \mathrm{dL}$ and a creatinine level of $1.43 \mathrm{mg} / \mathrm{dL}$; however, serum electrolytes were within the normal range. Although serum flecainide levels were not analyzed, the history of flecainide ingestion together with the wide QRS complex on the ECG suggested flecainide-induced TdP. After IV administration of $80 \mathrm{mEq}$ of an $8.4 \%$ solution of sodium bicarbonate, the patient regained spontaneous circulation and the femoral pulse was palpable; CPR was therefore terminated.

A jugular venous catheter and a left femoral arterial-line catheter were prepared; moreover, an L-tube and Foley catheter were inserted. Gastric lavage was performed, followed by the administration of $50 \mathrm{~g}$ of activated charcoal. The patient's blood pressure was $87 / 44 \mathrm{mmHg}$, heart rate $104 \mathrm{BPM}$, and $\mathrm{SpO}_{2} 97 \%$. Continuous IV infusions of norepinephrine and heparin were started; sodium bicarbonate was also intravenously administered continuously at a rate of $45 \mathrm{mEq} / \mathrm{h}$. Additionally, $2 \mathrm{~g}$ of IV $\mathrm{MgSO}_{4}$ was administered, and a continuous IV infusion of amiodarone was initiated.

The patient was then transferred to the medical intensive care unit for ongoing observation. Upon arrival, various cardiac arrhythmias, including ventricular fibrillation, were noted (Figure 3). Unfortunately, there was no palpable spontaneous pulse. CPR was initiated together with an IV administration of $8 \mathrm{mg}$ of epinephrine and $8 \mathrm{mg}$ of atropine. After complete CPR, including three rounds of direct-current (DC) cardioversion for a total of 21 minutes, femoral pulsation became palpable and the CPR was terminated. Since ventricular arrhythmia was sustained after $\mathrm{CPR}$, lidocaine was administered first as a $60 \mathrm{mg}$ IV bolus and then as a continuous IV infusion at $3 \mathrm{mg} / \mathrm{h}$.

After stabilization, the ECG showed a normal ejection fraction (57\%) with no segmental wall motion abnormalities. Fifty hours 


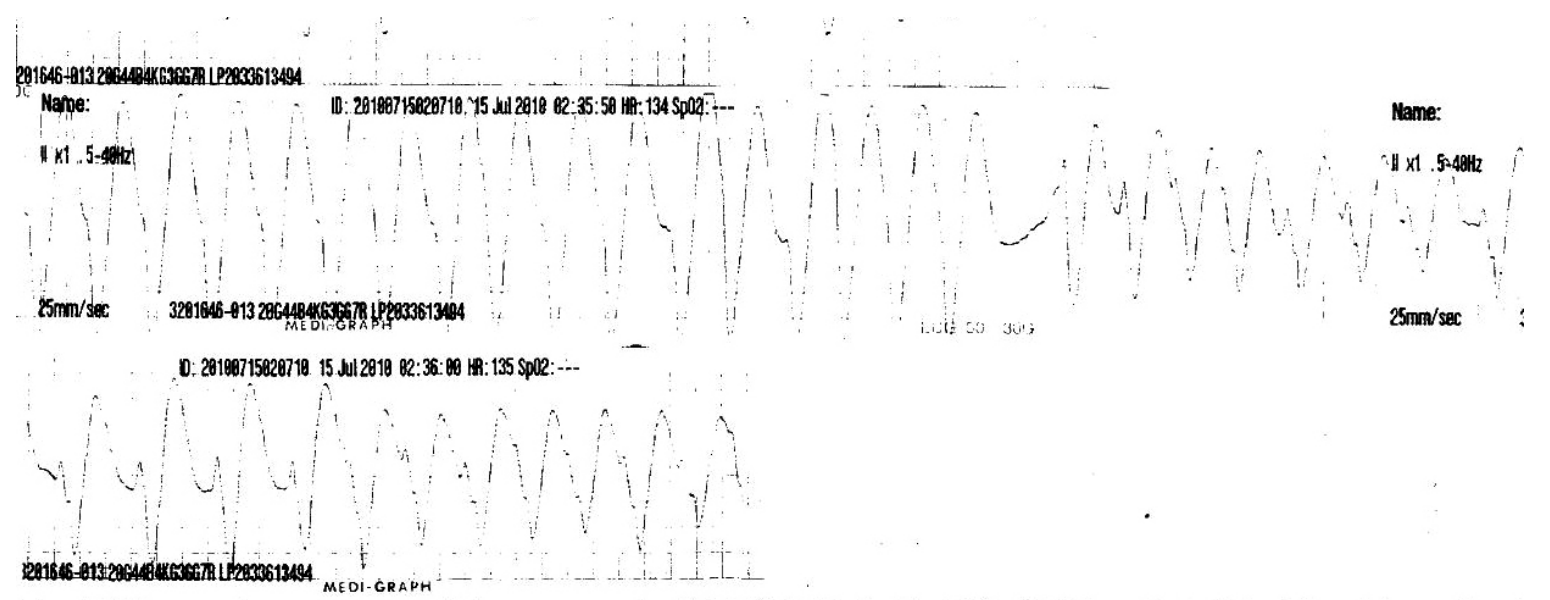

Figure 2. Electrocardiogram in the emergency department during cardiopulmonary resuscitation. The rhythm strip recording showed irregular, wide-complex tachycardia.

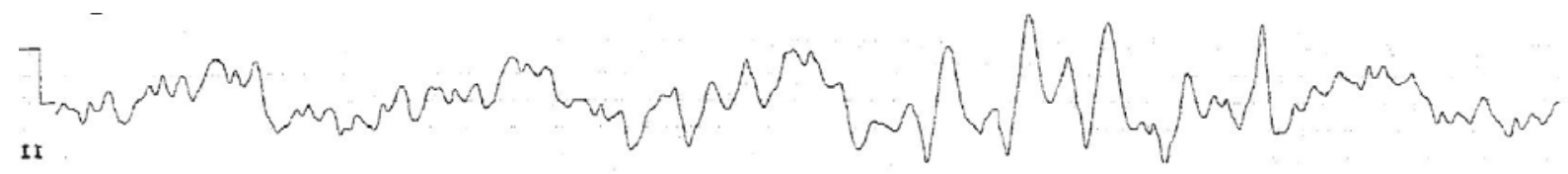

Figure 3. Electrocardiogram (ECG) after intensive care unit admission during cardiopulmonary resuscitation. The ECG showed ventricular fibrillation.

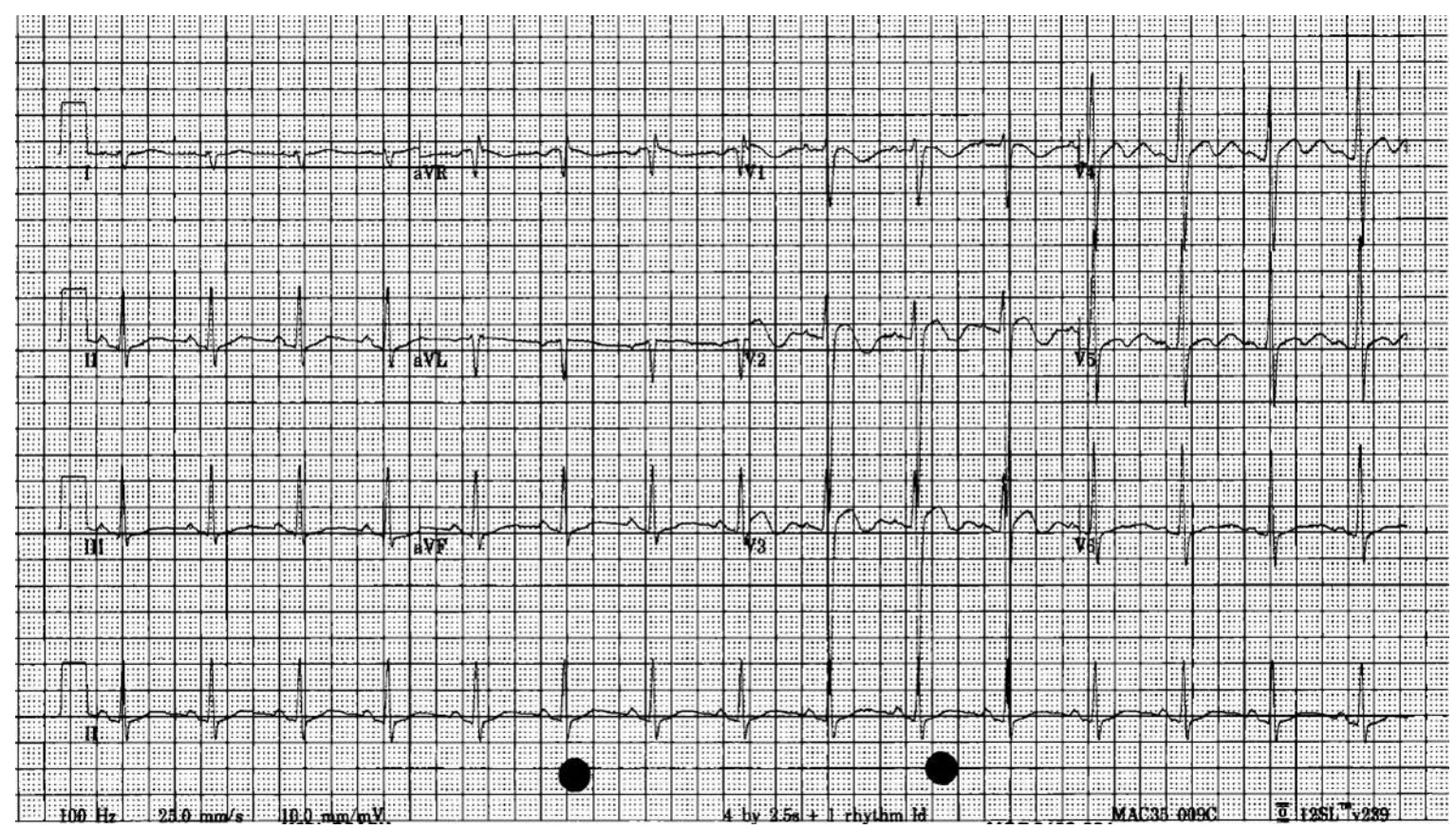

Figure 4. Electrocardiogram (ECG) during the 3rd day of hospitalization. The ECG showed a normal sinus rhythm. The QRS duration is 106 msec and corrected QT interval is $608 \mathrm{msec}$. 
post ingestion, the patient remained hemodynamically stable; the ECG displayed a sinus rhythm at a rate of 89 BPM and a prolonged QTc of $608 \mathrm{msec}$ (Figure 4). He was then extubated and finally, made a full recovery. He was discharged 7 days post admission with no evidence of end organ damage.

\section{Discussion}

Flecainide acetate is a Vaughan Williams class IC antiarrhythmic agent used to treat supraventricular and ventricular arrhythmias. It acts by blocking the fast inward sodium channel during phase 0 of the action potential, resulting in a marked depression of all major conduction pathways. Overdose symptoms include nausea, vomiting, hypotension, bradycardia, varying degrees of atrioventricular block, and tachyarrhythmia. Compared with other acute drug intoxications, class IC drugs overdoses are associated with a high mortality rate. ${ }^{1}$

Drugs that interact with their receptors more at faster heart rates are said to display use dependence. Class I antiarrhythmic drugs have frequency dependent effects on cardiac sodium channels, leading to greater reductions in $\mathrm{V}_{\max }$ of ventricular tissue at faster stimulation rates. CAST (Cardiac Arrhythmia Suppression Trial) reports have emphasized the potential deleterious or proarrhythmic risks of type I antiarrhythmic drug therapy. ${ }^{2}$ In particular, the rate-dependent conduction slowing associated with the flecainide has been implicated as a potential mechanism for drug proarrhythmia.

The risk of pro-arrhythmia was reported in patients with myocardial infarction, who were taking flecainide. Although flecainide suppresses conductivity, it may perpetuate reentry in patients with structural heart disease. Flecainide-induced proarrhythmia has rarely been reported in patients with no structural heart disease. Instead, it is most often found in patients taking other anti-arrhythmic drugs, having electrolyte disorders, or an atrial flutter with a 1:1 ventricular conduction. ${ }^{3}$ Since we did not analyze flecainide blood levels, we could not confirm if this was a case of flecainide toxicity. However, since toxicity is suggested when there is a $50 \%$ increase in the duration of QRS or $30 \%$ increase in the prolongation of the PR interval, it is likely. ${ }^{4}$

Here, we report a patient with flecainide-associated bradycardia-dependent TdP. In this patient, the onset of TdP showed a marked QT prolongation beyond $600 \mathrm{msec}$, and ectopic beats of the identical right bundle branch block on both occasions. Flecainide may lengthen the QT interval, mainly causing QRS broadening, as it lacks any significant effect on repolarization. Therefore, it is not believed to induce $\mathrm{TdP}$ alone or polymorphic ventricular tachycardia by lengthening QT. Indeed, cases of flecainide-induced TdP are rare and generally associated with other anti-arrhythmic drugs and/or electrolyte disorders. There are only a few published studies in which tachyarrhythmia is directly linked with flecainide in the absence of other associated triggering factors. ${ }^{5}$

Flecainide toxicity induce sinus node dysfunction, atrioventricular block, and QT prolongation. QT prolongation in flecainide toxicity is thought to be because of IKr channels in the ventricular myocytes.

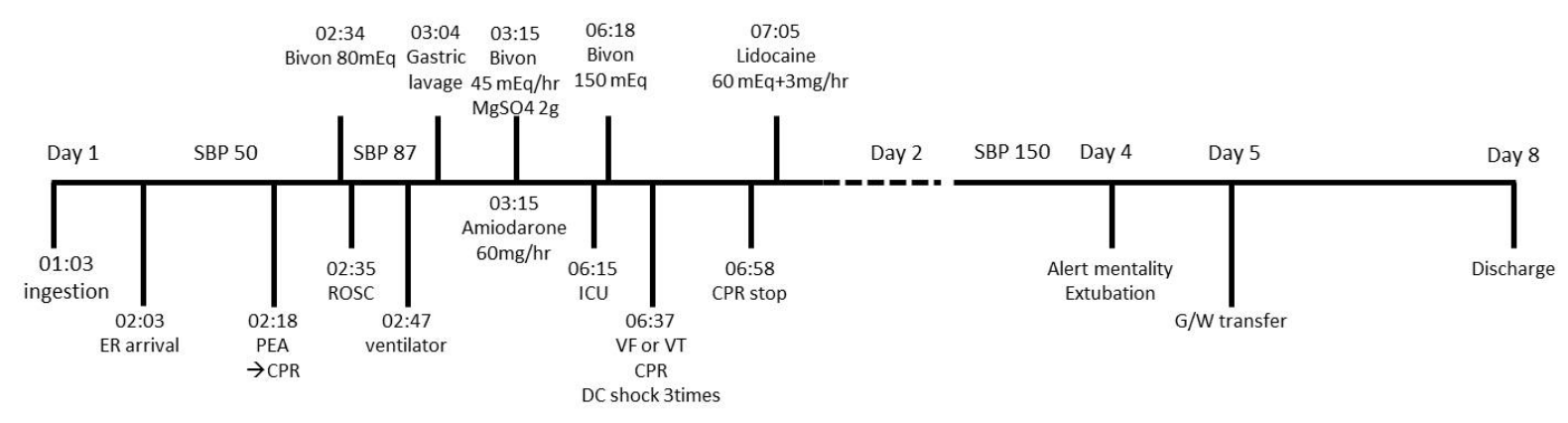

Wide and irregular heart rhythm

Sinus rhythm

Figure 5. Timeline representation of events. After the administration of sodium bicarbonate, amiodarone, magnesium sulfate, and lidocaine, the wide and irregular heart rhythm was restored to a sinus rhythm.

CPR. cardiopulmonary resuscitation, DC. direct current; ER, emergency room; G/W, general ward; ICU, intensive care unit; PEA, pulseless electrical activity; ROSC, return of spontaneous circulation; SBP, systolic blood pressure; VF, ventricular fibrillation; VT, ventricular tachycardia. 
Absorption from the gastrointestinal tract is reasonably rapid, with maximum concentration occurring after 3-4 hours post drug ingestion. ${ }^{4}$ Flecainide is eliminated, largely unchanged, by the kidney, and is metabolized to some degree by the hepatic cytochrome P450 2D6. It also has a long elimination half-life (7 to 24 hours), which makes the treatment of a severe overdose more difficult. $^{6}$

The primary treatment of an acute flecainide overdose should be aimed at reducing gastrointestinal absorption via gastric lavage and activated charcoal administration. Other available measures of detoxification include forced diuresis, hemofiltration, and hemoperfusion. However, these procedures have limitations: forced diuresis may exacerbate flecainide-induced cardiac insufficiency; hemofiltration may not remove flecainide effectively as the drug is tightly bound to proteins; and finally, hemoperfusion may result in hypocalcaemia, which would further prolong the QTc interval.

Administration of a hypertonic sodium bicarbonate solution appears to improve the hemodynamic instability resulting from flecainide toxicity. As illustrated in this case, the benefits can be dramatic and occur within minutes, despite the fact that the mechanism of action is complex and involves the combination of an increase of intracellular sodium and $\mathrm{pH}$, resulting in the displacement of flecainide from the sodium channels. ${ }^{8}$ However, there is no recommended dose of sodium bicarbonate. One common practice is to use a bolus of 50 to $100 \mathrm{mEq}$ of hypertonic sodium bicarbonate solution, targeting a $\mathrm{pH}$ value of 7.5 to 7.55 . There are several case reports demonstrating that intensive sodium bicarbonate therapy improves survival without resorting to ECMO. In the case reported herein, the total dose of bicarbonate used was above $500 \mathrm{mEq}$.

There are case reports showing that anti-arrhythmic agents have been used to terminate flecainide-induced arrhythmias; moreover, in several case reports, sinus rhythm was restored soon after the administration of lidocaine. Although lidocaine is also a sodium channel blocker, it is postulated that its fast-on/fast-off kinetics allow it to compete for the sodium channel, hence reversing the toxicity of other more potent sodium channel blockers (such as the class IC agents). ${ }^{10}$ Other case reports have identified IV amiodarone or $\mathrm{MgSO}_{4}$ as the principle agent responsible for terminating flecainide-induced ventricular arrhythmias. ${ }^{11,12}$
Because hypotension can develop rapidly after a flecainide overdose, hepatic and renal blood flow is reduced. Measures to maintain vital organ perfusion will enhance flecainide clearance and its redistribution to other body tissues. One method to provide the hemodynamic support necessary to allow flecainide clearance and redistribution is ECMO. Yasui, et al. ${ }^{13}$ showed that this technique allowed flecainide clearance and redistribution to continue in one patient with flecainide overdose, reducing the half-life of the drug to six hours. Since the use of ECMO is accompanied by complications including coagulopathies (requiring blood product support), hemorrhage at the cannulation point, and femoral nerve palsy, it should be used cautiously.

Although there is no standard treatment for flecainide overdose, the case reported here demonstrates that an intensive pharmacological therapy, including the administration of sodium bicarbonate, amiodarone, $\mathrm{MgSO}_{4}$, and lidocaine, can rescue patients from flecainide toxicity with no recourse to extracorporeal therapy.

\section{References}

1) Auzinger GM, Scheinkestel CD. Successful extracorporeal life support in a case of severe flecainide intoxication. Crit Care Med. 2001;29:887-890.

2) The Caridac Arrhythmia Suppression Trial (CAST) Investigators: Preliminary report: Effect of encainide and flecainide on mortality in a randomized trial of arrhythmia suppression after myocardial infarction. NEngl J Med. 1989;321:406-412.

3) Kim HS, Pak HN, Park JS, Kim SS. Flecainide-associated bradycardia-dependent torsade de pointes: another potential mechanism of proarrhythmia. Pacing Clin Electrophysiol. 2013;36:e84-e86.

4) Brazil E, Bodiwala GG, Bouch DC. Fatal flecainide intoxication. $J$ Accid Emerg Med. 1998:15:423-425.

5) Nogales Asensio JM, Moreno Sanchez N, Doncel Vecino LJ, Villar Mariscal C, Lopez-Minguez JR, Merchan Herrera A. Torsade-depointes in a patient under flecainide treatment, an unusual case of proarrhythmicity. Int J Cardiol. 2007;114:E65-67.

6) Aliot E, Capucci A, Crijns HJ, Goette A, Tamargo J. Twenty-five years in the making: flecainide is safe and effective for the management of atrial fibrillation. Europace. 2011:13:161-173.

7) Hanley NA, Bourke JP, Gascoigne AD. Survival in a case of lifethreatening flecainide overdose. Intensive Care Med. 1998;24:740-742. 
8) Bond R, Augustine D, Walker S. Iatrogenic flecainide toxicity. Heart. 2010;96:2048-2049.

9) Devin R, Garrett P, Anstey C. Managing cardiovascular collapse in severe flecainide overdose without recourse to extracorporeal therapy. Emerg Med Australas. 2007;19:155-159.

10) Bauman JL, Gallastegui J, Tanenbaum SR, Hariman RJ. Flecainide-induced sustained ventricular tachycardia successfully treated with lidocaine. Chest. 1987;92:573-575.
11) Siegers A, Board PN. Amiodarone used in successful resuscitation after near-fatal flecainide overdose. Resuscitation. 2002;53:105-108.

12) Williamson DG, Sinha A, Frost I, Singh VK. Management of persistent wide QRS in flecainide overdose with magnesium sulphate. Emerg Med J. 2010;27:487-488.

13) Yasui RK, Culclasure TF, Kaufman D, Freed CR. Flecainide overdose: is cardiopulmonary support the treatment? Ann Emerg Med. 1997;29:680-682. 